\title{
A RENEWAL-PROCESS-TYPE EXPRESSION FOR THE MOMENTS OF INVERSE SUBORDINATORS
}

\author{
ANDREAS NORDVALL LAGERÅS, * Stockholm University
}

\begin{abstract}
We define an inverse subordinator as the passage times of a subordinator to increasing levels. It has previously been noted that such processes have many similarities to renewal processes. Here we present an expression for the joint moments of the increments of an inverse subordinator. This is an analogue of a result for renewal processes. The main tool is a theorem on processes which are both renewal processes and Cox processes.
\end{abstract}

Keywords: Subordinator; passage time; renewal theory; Cox process; local time

2000 Mathematics Subject Classification: Primary 60K05

Secondary 60G51; 60G55; 60E07

\section{Introduction}

Subordinators are nondecreasing processes with independent and stationary increments. The corresponding processes in discrete time are the partial-sum processes with positive, independent, and identically distributed summands. Renewal processes can be considered to be passage times of partial-sum processes to increasing levels. Analogously, we can define a process by the passage times of a subordinator. We call such a process an inverse subordinator.

The inverse subordinators appear in diverse areas of probability theory. As Bertoin [2] noted, the local times of a large class of well-behaved Markov processes are really inverse subordinators, and any inverse subordinator is the local time of some Markov process. It is well known (see Karatzas and Shreve [8]) that the local time of a Brownian motion is the inverse of a $\frac{1}{2}$-stable subordinator. Inverses of $\alpha$-stable subordinators with $0<\alpha<1$ arise as limiting processes of occupation times of Markov processes (see Bingham [4]). Some recent applications of inverse subordinators in stochastic models can be found in [7], [10], and [14]. Kaj and Martin-Löf [7] considered superposition and scaling of inverse subordinators with applications in queueing theory, Kozlova and Salminen [10] used diffusion local time as input in a so-called storage process, and Winkel [14] used inverse subordinators in financial modelling.

In this paper, we study some general distributional properties of inverse subordinators, using renewal theory and some theory about Cox processes. In particular, we find an expression for the joint moments of their increments. Other results for inverse subordinators analogous to those in renewal theory have been proved (see [3] and [13]).

Some well-known results on subordinators and infinitely divisible distributions on the positive real line are given in Section 2. Section 3 introduces the inverse subordinators and hints that they may have properties similar to the renewal processes. In Section 4, the main result is

Received 28 February 2005; revision received 4 July 2005.

* Postal address: Department of Mathematics, Stockholm University, SE-10691 Stockholm, Sweden.

Email address: andreas@math.su.se 
given: an expression for the joint moments of the increments of an inverse subordinator. This is proved using a representation of the class of point processes that are both Cox processes and renewal processes. With this representation, we can also give an alternative proof of the fact that inverse subordinators can be delayed to be given stationary increments (see [13]). We also provide a bound of the upper tail of the marginal distribution of an inverse subordinator. Finally, in Section 5 we exemplify the results with three types of inverse subordinator.

\section{Some basic facts about subordinators}

The following results on infinitely divisible distributions and Lévy processes can be found in [12]. Let $\left\{Y_{t}\right\}$ be a Lévy process, i.e. a stochastic process in continuous time with $Y_{0}=0$ and stationary and independent increments. The distribution $F$ of $Y_{1}$ is necessarily infinitely divisible, i.e. for all $n \in \mathbb{N}$ there is a distribution $F_{n}$ such that $F_{n}^{\star n}=F$. Here $F_{n}^{\star n}$ is the $n$-fold convolution of $F_{n}$. The converse is also true: given an infinitely divisible distribution $F$, there is a Lévy process $\left\{Y_{t}\right\}$ such that the distribution of $Y_{1}$ is $F$. Define $F^{\star t}$ for positive, noninteger $t$ by $F^{\star t}(x)=\mathrm{P}\left(Y_{t} \leq x\right)$. We recognize that $F_{n}=F^{\star 1 / n}$.

If we restrict $F$ to be a distribution on $\mathbb{R}_{+}$then the increments of $\left\{Y_{t}\right\}$ are all nonnegative. Lévy processes with nonnegative increments are called subordinators. It is well known that the Laplace-Stieltjes transform of $F^{\star t}$, where $F$ is an infinitely divisible distribution on $\mathbb{R}_{+}$, can be written

$$
\widehat{F^{\star t}}(u)=\int_{0}^{\infty} \mathrm{e}^{-u x} F^{\star t}(\mathrm{~d} x)=\mathrm{e}^{-t \psi(u)}=\widehat{F}(u)^{t},
$$

where $\psi(u)$ is called the Lévy exponent and can be written in the following form:

$$
\psi(u)=\delta u+\int_{0}^{\infty}\left(1-\mathrm{e}^{-u x}\right) v(\mathrm{~d} x) .
$$

Here, $\delta \geq 0$ is called the drift and $v(\mathrm{~d} x)$ is called the Lévy measure. If $Y_{1}$ has drift $\delta$ then $Y_{1}-\delta$ has drift 0 . If $\int_{0}^{\infty} v(\mathrm{~d} x)<\infty$ then $\left\{Y_{t}\right\}$ is a compound Poisson process, with drift if $\delta>0$, and thus only makes a finite number of jumps in any finite interval. We call a function $\pi$ the Lévy density if $\nu(A)=\int_{A} \pi(x) \mathrm{d} x$. If we define $\mu=\mathrm{E}\left[Y_{1}\right]$, then $\mu=\delta+\int_{0}^{\infty} x v(\mathrm{~d} x)$. Since $\psi^{\prime}(u)=\delta+\int_{0}^{\infty} \mathrm{e}^{-u x} x v(\mathrm{~d} x)$, we have

$$
\psi^{\prime}(0)=\mu \quad \text { and } \quad \psi^{\prime}(u) \searrow \delta \quad \text { as } u \nearrow \infty .
$$

Some parts of the reasoning in the following sections do not apply to compound Poisson processes without drift. Therefore, we will henceforth, albeit somewhat artificially, exclude the compound Poisson processes without drift when referring to subordinators.

\section{Inverse subordinators and renewal processes}

It is advantageous to recall some results on renewal processes before a more thorough study of subordinators and their inverses. Let $X_{2}, X_{3}, \ldots$ be a sequence of independent and identically distributed (strictly) positive random variables with distribution $F$, and $X_{1}$ a positive random variable with distribution $H$, independent of $X_{2}, X_{3}, \ldots$ Let $S_{0}=0$ and $S_{n}=\sum_{k=1}^{n} X_{k}$; we call $\left\{S_{n}\right\}$ a partial-sum process. Given a partial-sum process, we define the renewal process with interarrival distribution $F$ by $N_{t}=\min \left(n \in \mathbb{N}: S_{n}>t\right)-1$. The ' -1 ' in the definition comes from the fact that we do not want to count the renewal at the origin, as is sometimes done. If $F=H$ then $\left\{N_{t}\right\}$ is called an ordinary renewal process. 
It is well known that $\left\{N_{t}\right\}$ has stationary increments if and only if

$$
H(x)=\frac{1}{\mu} \int_{0}^{x}(1-F(y)) \mathrm{d} y,
$$

where $\mu=\mathrm{E}\left[X_{2}\right]=\int_{0}^{\infty}(1-F(x)) \mathrm{d} x$ and is necessarily finite (see [5]). Then we also have

$$
\mathrm{E}\left[X_{1}\right]=\frac{\mathrm{E}\left[X_{2}^{2}\right]}{2 \mu},
$$

and the Laplace-Stieltjes transform of $H$ is

$$
\widehat{H}(s)=\frac{1}{\mu s}(1-\widehat{F}(s)) .
$$

We note, as in [3], that subordinators are continuous-time analogues of partial-sum processes. A Lévy process sampled at equidistant time points, for example $Y_{n}=\sum_{k=1}^{n}\left(Y_{k}-Y_{k-1}\right)$ when the time points are the integers, does produce a partial-sum process with an infinitely divisible $F$. As the renewal processes are integer-valued inverses to partial-sum processes, an inverse of a subordinator could be expected to have some properties similar to renewal processes. Given a subordinator $\left\{Y_{t}\right\}$, we define $\tau_{t}=\inf \left(\tau>0: Y_{\tau}>t\right)$ and call the process $\left\{\tau_{t}\right\}_{t \geq 0}$ the inverse subordinator.

The properties of the paths of $\left\{\tau_{t}\right\}$ differ depending on $\left\{Y_{t}\right\}$. Let us first consider a compound Poisson process $\left\{Y_{t}\right\}$ with drift $\delta>0$. Since a jump in $\left\{Y_{t}\right\}$ corresponds to a flat period in its inverse, $\left\{\tau_{t}\right\}$ alternates between linearly increasing with slope $1 / \delta$ for exponential periods of time and being constant for periods of time with lengths drawn from the compounding distribution, with all these periods having independent lengths. It is more tricky when $\left\{Y_{t}\right\}$ is not a compound Poisson process and the drift is zero. Owing to the fact that $\left\{Y_{t}\right\}$ in this case makes an infinite number of jumps in any finite interval, the trajectories of $\left\{\tau_{t}\right\}$ are continuous and, considered as distribution functions of measures, singular with respect to the Lebesgue measure (see [6, p. 39]).

Now we will show that $\left\{\tau_{t}\right\}$ can be arbitrarily closely approximated by a scaled renewal process. For any $c>0$, let $\left\{Y_{t}^{c}\right\}$ be defined by $Y_{t}^{c}=Y_{t / c}$. Note that $\left\{Y_{t}^{c}\right\}$ is a subordinator with $Y_{1}^{c} \sim F^{\star 1 / c}$. Also define the renewal process $N_{t}^{c}=\min \left(n \in \mathbb{N}: Y_{n}^{c}>t\right)-1$. Since

$$
\begin{aligned}
c \tau_{t} & =c \inf \left(\tau>0: Y_{\tau}>t\right) \\
& =\inf \left(c \tau>0: Y_{\tau}>t\right) \\
& =\inf \left(\tau>0: Y_{\tau / c}>t\right) \\
& =\inf \left(\tau>0: Y_{\tau}^{c}>t\right) \\
& \geq \min \left(n \in \mathbb{N}: Y_{n}^{c}>t\right)-1 \\
& \geq \inf \left(\tau>0: Y_{\tau}^{c}>t\right)-1 \\
& =c \tau_{t}-1,
\end{aligned}
$$

we obtain

$$
\tau_{t}=\frac{1}{c} N_{t}^{c}+r_{t}, \quad \text { where } 0 \leq r_{t} \leq \frac{1}{c},
$$

and the approximation becomes arbitrarily good as $c \rightarrow \infty$. This result suggests that the inverse subordinators may have some properties similar to renewal processes. That this is in fact true will be shown in Section 4. 
An important function in the theory of renewal processes is the so-called renewal function $V(t)=\mathrm{E}\left[N_{t}\right]$. We note that for an ordinary renewal process $V(t)=\sum_{k=1}^{\infty} F^{\star k}(t)$, and for a stationary renewal process $V(t)=t / \mu$. If there is a function $v$ such that $V(t)=\int_{0}^{t} v(s) \mathrm{d} s$, then $v$ is called the renewal density. If the renewal process had been defined to also count the renewal at the origin, then the renewal function would be $V(t)+1$. We can also define a renewal function for the inverse subordinator. Given an inverse subordinator $\left\{\tau_{t}\right\}$, we define its renewal function $U$ by $U(t)=\mathrm{E}\left[\tau_{t}\right]$. The renewal function can be expressed as follows:

$$
U(t)=\mathrm{E}\left[\tau_{t}\right]=\int_{0}^{\infty} \mathrm{P}\left(\tau_{t}>x\right) \mathrm{d} x=\int_{0}^{\infty} \mathrm{P}\left(Y_{x} \leq t\right) \mathrm{d} x=\int_{0}^{\infty} F^{\star x}(t) \mathrm{d} x .
$$

The expression on the right-hand side might be hard to evaluate, but its Laplace-Stieltjes transform is easily calculated as follows:

$$
\begin{aligned}
\widehat{U}(s) & =\int_{0}^{\infty} \mathrm{e}^{-s t} \int_{0}^{\infty} F^{\star x}(\mathrm{~d} t) \mathrm{d} x \\
& =\int_{0}^{\infty} \widehat{F}(s)^{x} \mathrm{~d} x \\
& =\int_{0}^{\infty} \mathrm{e}^{-x \psi(s)} \mathrm{d} x \\
& =\frac{1}{\psi(s)} .
\end{aligned}
$$

Thus, there is a one-to-one correspondence between the renewal function and the distribution of $\left\{\tau_{t}\right\}$. This also correlates with the similar result for ordinary renewal processes and their renewal functions. Define the factorial power $n^{[k]}$ for $n, k \in \mathbb{N}$ as follows:

$$
n^{[k]}= \begin{cases}n(n-1) \cdots(n-k+1) & \text { for } n \geq k \geq 1 \\ 1 & \text { for } k=0 \\ 0 & \text { for } n<k, k \geq 1\end{cases}
$$

Given a renewal process and its renewal function, moments of all orders can be calculated as stated in the following proposition (see [5]).

Proposition 1. Let $\left\{N_{t}\right\}$ be a renewal process with interarrival distribution $F$, and let $V(t)=$ $\sum_{k=1}^{\infty} F^{\star k}(t)$. If $\left\{N_{t}\right\}$ is an ordinary renewal process then, for $s_{1}, \ldots, s_{n}$ and $t_{1}, \ldots, t_{n}$ such that $0 \leq s_{1}<t_{1} \leq s_{2}<\cdots<t_{n}$ and $k_{1}, \ldots, k_{n} \in \mathbb{N} \backslash\{0\}$ such that $k_{1}+\cdots+k_{n}=k$, we have

$$
\mathrm{E}\left[\prod_{i=1}^{n}\left(N_{t_{i}}-N_{s_{i}}\right)^{\left[k_{i}\right]}\right]=\prod_{i=1}^{n} k_{i} ! \int_{C} \prod_{j=1}^{k} V\left(\mathrm{~d} x_{j}-x_{j-1}\right)
$$

where

$C=\left\{x_{0}, \ldots, x_{k}: x_{0}=0, s_{i}<x_{k_{0}+\cdots+k_{i-1}+1}<\cdots<x_{k_{0}+\cdots+k_{i}} \leq t_{i}, i=1, \ldots, n, k_{0}=0\right\}$.

If $\left\{N_{t}\right\}$ is stationary, then the proposition also holds with the first factor of the right-most product in (5) replaced by $\mathrm{d} x_{1} / \mu$. 
Sketch of a proof of Proposition 1. We can write $N_{t}-N_{s}=\int_{(s, t]} N(\mathrm{~d} x)$ and

$$
\left(N_{t}-N_{s}\right)^{k}=\int_{(s, t]^{k}} \prod_{j=1}^{k} N\left(\mathrm{~d} x_{j}\right) .
$$

Note that $n^{k}$ is the number of $k$-tuples of integers from 1 to $n$, and $n^{[k]}$ is the number of $k$-tuples of integers such that no integers in the $k$-tuple are the same. Thus, we can write

$$
\left(N_{t}-N_{s}\right)^{[k]}=\int_{A} \prod_{j=1}^{k} N\left(\mathrm{~d} x_{j}\right),
$$

where $A=\left\{\left(x_{1} \ldots x_{k}\right) \in(s, t]^{k}: x_{p} \neq x_{q}\right.$ for $\left.p \neq q\right\}$. The renewal property is used in the following expression:

$$
\begin{aligned}
\mathrm{E}\left[\prod_{j=1}^{k} N\left(\mathrm{~d} x_{j}\right)\right] & =\mathrm{P}\left(N\left(\mathrm{~d} x_{1}\right)=1, \ldots, N\left(\mathrm{~d} x_{k}\right)=1\right) \\
& =\mathrm{P}\left(N\left(\mathrm{~d} x_{(1)}\right)=1\right) \prod_{j=2}^{k} \mathrm{P}\left(N\left(\mathrm{~d} x_{(j)}\right)=1 \mid N\left(\mathrm{~d} x_{(j-1)}\right)=1\right) \\
& =\mathrm{P}\left(N\left(\mathrm{~d} x_{(1)}\right)=1\right) \prod_{j=2}^{k} V\left(\mathrm{~d} x_{(j)}-x_{(j-1)}\right) .
\end{aligned}
$$

The first factor is equal to $V\left(\mathrm{~d} x_{(1)}\right)$ in the ordinary case and is equal to $\mathrm{d} x_{(1)} / \mu$ in the stationary case. Let

$$
\begin{aligned}
A_{i} & =\left\{\left(y_{i 1}, \ldots, y_{i k_{i}}\right) \in\left(s_{i}, t_{i}\right]^{k_{i}}: y_{i p} \neq y_{i q} \text { for } p \neq q\right\}, \\
B_{i} & =\left\{\left(y_{i 1}, \ldots, y_{i k_{i}}\right): s_{i}<y_{i 1}<\cdots<y_{i k_{i}} \leq t_{i}\right\} .
\end{aligned}
$$

Thus, in the ordinary case,

$$
\begin{aligned}
\mathrm{E}\left[\prod_{i=1}^{n}\left(N_{t_{i}}-N_{s_{i}}\right)^{\left[k_{i}\right]}\right] & =\mathrm{E}\left[\prod_{i=1}^{n} \int_{A_{i}} \prod_{j=1}^{k_{i}} N\left(\mathrm{~d} y_{i j}\right)\right] \\
& =\mathrm{E}\left[\prod_{i=1}^{n} k_{i} ! \int_{B_{i}} \prod_{j=1}^{k_{i}} N\left(\mathrm{~d} y_{i j}\right)\right] \\
& =\prod_{i=1}^{n} k_{i} ! \mathrm{E}\left[\int_{C} \prod_{l=1}^{k} N\left(\mathrm{~d} x_{l}\right)\right] \\
& =\prod_{i=1}^{n} k_{i} ! \int_{C} \prod_{l=1}^{k} V\left(\mathrm{~d} x_{l}-x_{l-1}\right) .
\end{aligned}
$$

\section{Inverse subordinators and Cox processes}

An expression similar to (5) for the moments of $\left\{\tau_{t}\right\}$ can be obtained. First recall the definition of a Cox process. Let $\left\{N_{t}^{\lambda}\right\}$ be an inhomogeneous Poisson process on $\mathbb{R}_{+}$with 
intensity measure $\lambda$. Let $\Lambda$ be a random measure on $\mathbb{R}_{+}$. If the point process $\left\{M_{t}\right\}$ has the distribution of $\left\{N_{t}^{\lambda}\right\}$ conditional on $\Lambda=\lambda$, then $\left\{M_{t}\right\}$ is called a Cox process directed by $\Lambda$. Note that if $\left\{\widetilde{N}_{t}\right\}$ is a Poisson process with constant intensity equal to one and is independent of $\Lambda$, then $M_{t} \stackrel{\mathrm{D}}{=} \widetilde{N}(\Lambda((0, t]))$, where $\stackrel{\mathrm{D}}{=}$, denotes equality in distribution, and $\left\{M_{t}\right\}$ can be considered to be a homogeneous Poisson process subjected to a random time change by the random function $\Lambda((0, t])$. The interpretation of the Cox process as a time-changed Poisson process also describes how the points of the Cox process can be obtained from the points of the Poisson process. If we let $K(t)$ be the inverse of $\Lambda((0, t])$ and $t_{1}, t_{2}, \ldots$ are the points of $\left\{\tilde{N}_{t}\right\}$, then $K\left(t_{1}\right), K\left(t_{2}\right), \ldots$ are the points of $\left\{M_{t}\right\}$.

Also define a slight generalization of the inverse subordinators. Let $\widetilde{Y}_{0}$ have the distribution $G$ on $\mathbb{R}_{+}$and be independent of the subordinator $\left\{Y_{t}\right\}$ with $Y_{1} \sim F$. Define the process $\left\{\tilde{Y}_{t}\right\}$ by $\widetilde{Y}_{t}=Y_{t}+\widetilde{Y}_{0}$. Let $\tau_{t}=\inf \left(\tau>0: \widetilde{Y}_{\tau}>t\right)$, and call the process $\left\{\tau_{t}\right\}_{t \geq 0}$ a general inverse subordinator. If $\widetilde{Y}_{0} \equiv 0$ then we call $\left\{\tau_{t}\right\}$ an ordinary inverse subordinator.

We will see in Proposition 4 , below, that $\widetilde{Y}_{0}$ can be chosen so that the general inverse subordinator $\left\{\tau_{t}\right\}$ has stationary increments if $\mu=\mathrm{E}\left[Y_{1}\right]<\infty$. The following proposition is by Kingman [9] and Grandell [6].

Proposition 2. The Cox process $\left\{M_{t}\right\}$ directed by $\Lambda$ is a renewal process if and only if $\Lambda((s, t])=\tau_{t}-\tau_{s}$ for all $t>s$, where $\left\{\tau_{t}\right\}$ is a general inverse subordinator.

We will only prove the easier, 'if' part of this proposition, which is the only part to be used in Theorem 1.

Proof of Proposition 2. We note that $\widetilde{Y}_{t}$ is the inverse of $\Lambda((0, t])=\tau_{t}$. If we use the representation of $\left\{M_{t}\right\}$ as a time-changed Poisson process $\left\{\widetilde{N}_{t}\right\}$ with intensity one, then the points of $\left\{M_{t}\right\}$ are $\widetilde{Y}\left(t_{1}\right), \widetilde{Y}\left(t_{2}\right), \ldots$, where $t_{1}, t_{2}, \ldots$ are the points of $\left\{\widetilde{N}_{t}\right\}$. Since $\left\{Y_{t}\right\}$ is a subordinator, $\widetilde{Y}\left(t_{1}\right), \widetilde{Y}\left(t_{2}\right)-\widetilde{Y}\left(t_{1}\right), \widetilde{Y}\left(t_{3}\right)-\widetilde{Y}\left(t_{2}\right), \ldots$ are independent and $\widetilde{Y}\left(t_{2}\right)-\widetilde{Y}\left(t_{1}\right), \widetilde{Y}\left(t_{3}\right)-\widetilde{Y}\left(t_{2}\right), \ldots$ are furthermore equally distributed. Thus, $\left\{M_{t}\right\}$ is a renewal process.

We can say more about the interarrival distribution of $\left\{M_{t}\right\}$. Let

$$
Z=\tilde{Y}\left(t_{2}\right)-\tilde{Y}\left(t_{1}\right)=Y\left(t_{2}\right)-Y\left(t_{1}\right)
$$

and let $\varepsilon \sim \operatorname{Exp}(1)$, independent of $\left\{Y_{t}\right\}$. We obtain $Z=Y\left(t_{2}\right)-Y\left(t_{1}\right) \stackrel{\mathrm{D}}{=} Y\left(t_{2}-t_{1}\right) \stackrel{\mathrm{D}}{=} Y(\varepsilon)$, so the interarrival distribution of $\left\{M_{t}\right\}$ is thus compound exponential. The Laplace-Stieltjes transform of the distribution of $Z$ is given by

$$
\widehat{F}_{Z}(s)=\mathrm{E}\left[\mathrm{e}^{-s Z}\right]=\mathrm{E}\left[\mathrm{E}\left[\mathrm{e}^{-s Y(\varepsilon)} \mid \varepsilon\right]\right]=\mathrm{E}\left[\mathrm{e}^{-\psi(s) \varepsilon}\right]=\frac{1}{1+\psi(s)},
$$

where $\psi(s)$ is the Lévy exponent of $Y_{1}$. We now have the tools to prove the main result.

Theorem 1. Let $\left\{\tau_{t}\right\}$ be an ordinary inverse subordinator with renewal function $U(t)$. Then, for $s_{1}, \ldots, s_{n}$ and $t_{1}, \ldots, t_{n}$ such that $0 \leq s_{1}<t_{1} \leq s_{2}<\cdots<t_{n}$ and $k_{1}, \ldots, k_{n} \in \mathbb{N} \backslash\{0\}$ such that $k_{1}+\cdots+k_{n}=k$, we have

$$
\mathrm{E}\left[\prod_{i=1}^{n}\left(\tau_{t_{i}}-\tau_{s_{i}}\right)^{k_{i}}\right]=\prod_{i=1}^{n} k_{i} ! \int_{C} \prod_{j=1}^{k} U\left(\mathrm{~d} x_{j}-x_{j-1}\right),
$$

where $C$ is as in Proposition 1. If $\left\{\tau_{t}\right\}$ is stationary then the theorem also holds with the change that the first factor of the right-most product in (7) is replaced by $\mathrm{d} x_{1} / \mu$, but with the same $U$ in the remaining factors as the ordinary inverse subordinator. 
Proof. Define the random measure $\Lambda$ on $\mathbb{R}_{+}$by $\Lambda((s, t])=\tau_{t}-\tau_{s}$, for all $t>s \in \mathbb{R}_{+}$, and let $\left\{M_{t}\right\}$ be the Cox process directed by $\Lambda$. By Proposition $2,\left\{M_{t}\right\}$ is also a renewal process. Write $V(t)$ for its renewal function. Then

$$
V(t)=\mathrm{E}\left[M_{t}\right]=\mathrm{E}\left[\mathrm{E}\left[M_{t} \mid \tau_{t}\right]\right]=\mathrm{E}\left[\tau_{t}\right]=U(t) .
$$

Thus, we can replace $V(t)$ by $U(t)$ in (5) when calculating the factorial moments of $\left\{M_{t}\right\}$. As noted in [5], the factorial moments of the Cox process coincide with the ordinary moments of its directing measure and, by the construction of the directing measure, the stated result follows.

A renewal theorem for the inverse subordinators can also be given following Bertoin [2, Theorem I.21].

Proposition 3. If $\mu<\infty$ then $U(t) \sim t / \mu$ as $t \rightarrow \infty$.

Proof. Let $\left\{M_{t}\right\}$ be a Cox process directed by $\left\{\tau_{t}\right\}$ as in Proposition 2, and $V(t)$ its renewal function. By (8), $V(t)=U(t)$. An application of the renewal theorem for renewal processes (see [5]) provides the desired result.

Similar to renewal processes, the inverse subordinators can be delayed to become stationary. This has been proved by different methods in [7] and [13]. We now state this result and provide a proof based on the connection with Cox processes.

Proposition 4. Let $\left\{\tau_{t}\right\}$ be a general inverse subordinator with $\widetilde{Y}_{0} \sim G, Y_{1}=\widetilde{Y}_{1}-\widetilde{Y}_{0} \sim F$, and $\mu=\mathrm{E}\left[Y_{1}\right]<\infty$, where

$$
\psi(s)=-\log \widehat{F}(s)=\delta s+\int_{0}^{\infty}\left(1-\mathrm{e}^{-s x}\right) v(\mathrm{~d} x)
$$

and

$$
G(x)= \begin{cases}\frac{1}{\mu}\left(\delta+\int_{0}^{x} \int_{y}^{\infty} v(\mathrm{~d} z) \mathrm{d} y\right) & \text { for } x \geq 0, \\ 0 & \text { for } x<0 .\end{cases}
$$

Then $\left\{\tau_{t}\right\}$ has stationary increments.

Proof. By [6, Theorem 1.4], a Cox process is stationary if and only if its directing measure $\Lambda$ has stationary increments. Therefore, it suffices to check that the Cox process $\left\{M_{t}\right\}$ directed by $\left\{\tau_{t}\right\}$ is stationary. Its interarrival distribution $F_{Z}$ is given by (6). The $X_{1}$ of $\left\{M_{t}\right\}$ can be decomposed into $X_{1} \stackrel{\text { D }}{=} \widetilde{Y}_{0}+Z$, with $\widetilde{Y}_{0}$ and $Z$ independent, since the inverse subordinator is delayed by a time $\widetilde{Y}_{0}$ during which it is constant and equal to zero. The Laplace-Stieltjes transform of the distribution $H$ of $X_{1}$ is $\widehat{H}(s)=\widehat{G}(s) \widehat{F}_{Z}(s)$, where

$$
\begin{aligned}
\widehat{G}(s) & =\frac{1}{\mu} \int_{0}^{\infty} \mathrm{e}^{-s x}\left(\delta+\int_{x}^{\infty} v(\mathrm{~d} y)\right) \mathrm{d} x \\
& =\frac{1}{\mu}\left(\frac{\delta}{s}+\int_{0}^{\infty} \int_{0}^{y} \mathrm{e}^{-s x} \mathrm{~d} x v(\mathrm{~d} y)\right) \\
& =\frac{1}{\mu s}\left(\delta+\int_{0}^{\infty}\left(1-\mathrm{e}^{-s y}\right) v(\mathrm{~d} y)\right) \\
& =\frac{\psi(s)}{\mu s}
\end{aligned}
$$


Combining (6) and (10), we obtain

$$
\widehat{H}(s)=\widehat{G}(s) \widehat{F}_{Z}(s)=\frac{\psi(s)}{\mu s} \frac{1}{1+\psi(s)}=\frac{1}{\mu s}\left(1-\widehat{F}_{Z}(s)\right) .
$$

By (3), $X_{1}$ thus has the right distribution to make $\left\{M_{t}\right\}$ stationary.

Let $W_{t}$ be the excess of the renewal process and Cox process $\left\{M_{t}\right\}$, i.e. the time from $t$ to the next point of the process. When $\left\{M_{t}\right\}$ is stationary, $W_{t} \stackrel{\mathrm{D}}{=} X_{1} \stackrel{\mathrm{D}}{=} \widetilde{Y}_{0}+Z$. The decomposition of the excess can be given the following interpretation. From any given time $t$, the inverse subordinator will remain constant for a period which has the distribution $G$. During this time no points in the Cox process will occur. After that time the inverse subordinator starts anew and the distribution to the next point in the point process is given by $F_{Z}$. In the stationary case, we do not have to know $G$ explicitly to calculate $\mathrm{E}\left[\widetilde{Y}_{0}\right]$; if we use (2), we obtain $\mathrm{E}\left[X_{1}\right]=\mathrm{E}\left[Z^{2}\right] / 2 \mathrm{E}[Z]$. Now, $\mathrm{E}\left[X_{1}\right]=\mathrm{E}\left[\tilde{Y}_{0}\right]+\mathrm{E}[Z]$ and, by straightforward calculation using, for example, (6), we obtain $\mathrm{E}[Z]=\mathrm{E}\left[Y_{1}\right]$ and $\mathrm{E}\left[Z^{2}\right]=\operatorname{var}\left(Y_{1}\right)+2 \mathrm{E}\left[Y_{1}\right]^{2}$. Collecting and rearranging yields $\mathrm{E}\left[\widetilde{Y}_{0}\right]=\operatorname{var}\left(Y_{1}\right) / 2 \mathrm{E}\left[Y_{1}\right]$.

The expression (7) may be hard to use in practice to calculate higher joint moments. Nonetheless, the results above show that the covariance of two increments of a stationary inverse subordinator is a simple expression in the renewal function. Let $\left\{\tau_{t}\right\}$ be stationary and let $U(t)$ denote the renewal function of the corresponding ordinary inverse subordinator. Also, let $0<r \leq s<t$. We obtain

$$
\begin{aligned}
\operatorname{cov}\left(\tau_{r}, \tau_{t}-\tau_{s}\right) & =\mathrm{E}\left[\tau_{r}\left(\tau_{t}-\tau_{s}\right)\right]-\mathrm{E}\left[\tau_{r}\right] \mathrm{E}\left[\tau_{t}-\tau_{s}\right] \\
& =\int_{0}^{r} \int_{s}^{t} U(\mathrm{~d} x-y) \frac{\mathrm{d} y}{\mu}-\frac{r}{\mu} \frac{t-s}{\mu} \\
& =\frac{1}{\mu} \int_{0}^{r}(U(t-y)-U(s-y)) \mathrm{d} y-\frac{r(t-s)}{\mu^{2}} .
\end{aligned}
$$

Now consider the particular case where $r=1, s=n \geq 1, t=n+1$, and $U$ has a density $u$ such that $U(t)=\int_{0}^{t} u(s) \mathrm{d} s$. Also assume, for simplicity, that $\mu=1$. Then the following approximation can be calculated:

$$
\operatorname{cov}\left(\tau_{1}, \tau_{n+1}-\tau_{n}\right)=\int_{0}^{1}(U(n+1-y)-U(n-y)) \mathrm{d} y-1 \approx u(n)-1 .
$$

Given the distribution of the subordinator $\left\{Y_{t}\right\}$, the distribution of its inverse is given by $\mathrm{P}\left(\tau_{t} \leq x\right)=\mathrm{P}\left(Y_{x}>t\right)$. It may still be hard to find a closed-form expression for this distribution function. The tail probabilities for the ordinary inverse subordinator can nonetheless be estimated. Only the case $\delta=0$ is interesting, since if the drift $\delta$ is positive then $Y_{x}-\delta x$ is nonnegative and, thus, $\mathrm{P}\left(Y_{x} \leq t\right)=\mathrm{P}\left(Y_{x}-\delta x \leq t-\delta x\right)=0$ for $x>t / \delta$. Let $s \geq 0$. Then we have

$$
\mathrm{P}\left(\tau_{t}>x\right)=\mathrm{P}\left(Y_{x} \leq t\right)=\mathrm{P}\left(\mathrm{e}^{-s Y_{x}} \geq \mathrm{e}^{-s t}\right) \leq \frac{\mathrm{E}\left[\mathrm{e}^{-s Y_{x}}\right]}{\mathrm{e}^{-s t}}=\mathrm{e}^{s t-x \psi(s)} .
$$

By (1), the last expression has a unique minimum as a function of $s$. If $x$ is large enough $(x>t / \mu)$, then the $s$ that minimizes the expression is nonzero and given by $s=\psi^{\prime-1}(t / x)$, where $\psi^{\prime-1}$ is the inverse of $\psi^{\prime}$. Thus, for large enough $x$,

$$
\mathrm{P}\left(\tau_{t}>x\right) \leq \exp \left(t \psi^{\prime-1}\left(\frac{t}{x}\right)-x \psi\left(\psi^{\prime-1}\left(\frac{t}{x}\right)\right)\right) .
$$


There is another result on the marginal distribution of $\left\{\tau_{t}\right\}$ that deserves mentioning. This result can be found in [7] and [11], but we give a short proof based on identifying Laplace transforms as probabilities.

Proposition 5. Let $\varepsilon_{s}$ be exponentially distributed with mean $1 / s$ and independent of $\left\{\tau_{t}\right\}$. Then the Laplace-Stieltjes transform of the distribution of $\tau\left(\varepsilon_{s}\right)$ is given by

$$
\mathrm{E}\left[\mathrm{e}^{-u \tau\left(\varepsilon_{s}\right)}\right]=1-\frac{u \widehat{G}(s)}{u+\psi(s)},
$$

where $\widehat{G}(s)$ is the Laplace-Stieltjes transform of the distribution of $\widetilde{Y}_{0}$.

Proof. Let $\widetilde{\varepsilon}_{u}$ be exponentially distributed with mean $1 / u$, and independent of $\varepsilon_{s}$ and $\left\{\tau_{t}\right\}$. We note that for a nonnegative random variable $X$ independent of $\widetilde{\varepsilon}_{u}, \mathrm{P}\left(\widetilde{\varepsilon}_{u} \geq X\right)=$ $\mathrm{E}\left[\mathrm{P}\left(\widetilde{\varepsilon}_{u} \geq X \mid X\right)\right]=\mathrm{E}\left[\mathrm{e}^{-u X}\right]$, the Laplace-Stieltjes transform of the distribution of $X$. Thus, we have, in the ordinary case,

$$
\begin{aligned}
\mathrm{E}\left[\mathrm{e}^{-u \tau\left(\varepsilon_{s}\right)}\right] & =\mathrm{P}\left(\widetilde{\varepsilon}_{u} \geq \tau\left(\varepsilon_{s}\right)\right) \\
& =\mathrm{P}\left(Y\left(\widetilde{\varepsilon}_{u}\right)>\varepsilon_{s}\right) \\
& =1-\mathrm{P}\left(\varepsilon_{s} \geq Y\left(\widetilde{\varepsilon}_{u}\right)\right) \\
& =1-\mathrm{E}\left[\mathrm{e}^{-s Y\left(\widetilde{\varepsilon}_{u}\right)}\right] \\
& =1-\mathrm{E}\left[\mathrm{E}\left[\mathrm{e}^{-s Y\left(\widetilde{\varepsilon}_{u}\right)} \mid \widetilde{\varepsilon}_{u}\right]\right] \\
& =1-\mathrm{E}\left[\mathrm{e}^{-\psi(s) \widetilde{\varepsilon}_{u}}\right] \\
& =1-\frac{u}{u+\psi(s)} .
\end{aligned}
$$

Likewise, in the general case,

$$
\begin{aligned}
\mathrm{E}\left[\mathrm{e}^{-u \tau\left(\varepsilon_{s}\right)}\right] & =1-\mathrm{P}\left(\varepsilon_{s} \geq \tilde{Y}\left(\widetilde{\varepsilon}_{u}\right)\right) \\
& =1-\mathrm{P}\left(\varepsilon_{s} \geq \widetilde{Y}\left(\widetilde{\varepsilon}_{u}\right) \mid \varepsilon_{s} \geq \widetilde{Y}_{0}\right) \mathrm{P}\left(\varepsilon_{s} \geq \widetilde{Y}_{0}\right) \\
& =1-\mathrm{P}\left(\varepsilon_{s} \geq Y\left(\widetilde{\varepsilon}_{u}\right)\right) \mathrm{P}\left(\varepsilon_{s} \geq \widetilde{Y}_{0}\right) \\
& =1-\frac{u \widehat{G}(s)}{u+\psi(s)},
\end{aligned}
$$

where we have used the memorylessness of the exponential distribution.

\section{Examples}

The $\alpha$-stable distribution on $\mathbb{R}_{+}$has Lévy exponent $\psi(s)=s^{\alpha}$ with $0<\alpha<1$. This gives a renewal density $u(t)=1 /\left(\Gamma(\alpha) t^{1-\alpha}\right)$, where $\Gamma(\cdot)$ is the gamma function, for the corresponding inverse stable subordinator by inverting (4). Theorem 1 thus confirms the moment expressions in [4] (see, for example, [4, Equation (18)]).

The main obstacle to the use of Theorem 1 is the possible difficulty in finding an expression for the renewal function. It is possible to find the renewal density not only for the inverse stable subordinator, but also for the inverses of subordinators with inverse Gaussian and gammadistributed increments. In these two cases it is also possible to delay the processes to obtain stationary versions, which is not possible in the stable case. 
For the inverse Gaussian distribution, with probability density

$$
f(x)=\frac{\delta}{\sqrt{2 \pi x^{3}}} \exp \left(\delta \gamma-\frac{1}{2}\left(\frac{\delta^{2}}{x}+\gamma^{2} x\right)\right), \quad \delta>0, \gamma>0,
$$

Lévy exponent

$$
\psi(s)=\delta \sqrt{\gamma^{2}+2 s}-\delta \gamma
$$

and Lévy density

$$
\pi(x)=\frac{\delta}{\sqrt{2 \pi x^{3}}} \exp \left(-\frac{\gamma^{2} x}{2}\right),
$$

we obtain, by (9), a probability density of the delay $\tilde{Y}_{0}$ by integrating $\pi$ (with $\mu=\psi^{\prime}(0)=\delta / \gamma$ ) as follows:

$$
g(t)=\frac{1}{\mu} \int_{t}^{\infty} \pi(x) \mathrm{d} x=\gamma \sqrt{\frac{2}{\pi t}} \exp \left(-\frac{\gamma^{2} t}{2}\right)-\gamma^{2} \operatorname{erfc}\left(\gamma \sqrt{\frac{t}{2}}\right) .
$$

Here, $\operatorname{erfc}(\cdot)$ is the complementary error function defined by $\operatorname{erfc}(t)=(2 / \sqrt{\pi}) \int_{t}^{\infty} \mathrm{e}^{-s^{2}} \mathrm{~d} s$. We note that the density does not depend on the parameter $\delta$. We obtain the renewal density $u(t)$ from its Laplace transform, which is equivalent to the Laplace-Stieltjes transform of $U(t)$, by rewriting (4) as follows:

$$
\begin{aligned}
\widehat{U}(s) & =\frac{1}{\psi(s)} \\
& =\frac{1}{\delta \sqrt{\gamma^{2}+2 s}-\delta \gamma} \\
& =\frac{\gamma}{2 \delta s}+\frac{1}{\delta \sqrt{\gamma^{2}+2 s}}+\frac{\gamma^{2}}{2 \delta s \sqrt{\gamma^{2}+2 s}} .
\end{aligned}
$$

By [1, Equations (29.3.1), (29.3.11), and (29.3.44)], this implies that

$$
u(t)=\frac{\gamma}{\delta}+\frac{1}{\delta \sqrt{2 \pi t}} \exp \left(-\frac{\gamma^{2} t}{2}\right)-\frac{\gamma}{2 \delta} \operatorname{erfc}\left(\gamma \sqrt{\frac{t}{2}}\right) .
$$

The estimate (11) gives

$$
\mathrm{P}\left(\tau_{t}>x\right) \leq \exp \left(-\frac{\delta^{2} x^{2}}{2 t}+\delta \gamma x-\frac{\gamma^{2} t}{2}\right) .
$$

For the gamma distribution we have probability density

$$
f(x)=\frac{\alpha^{\nu}}{\Gamma(\nu)} x^{\nu-1} \mathrm{e}^{-\alpha x}, \quad v>0, \alpha>0,
$$

Lévy exponent

$$
\psi(s)=v \log \left(1+\frac{s}{\alpha}\right)
$$

and Lévy density

$$
\pi(x)=\frac{v}{x} \mathrm{e}^{-\alpha x} .
$$


So, by (9), the density of the delay is

$$
g(t)=\alpha \mathrm{E}_{1}(\alpha t),
$$

where $\mathrm{E}_{1}$ is the exponential integral defined by $\mathrm{E}_{1}(t)=\int_{t}^{\infty} \mathrm{e}^{-s} \mathrm{~d} s / s$. As in the inverse Gaussian case, the density only depends on one parameter. The renewal density is also, in the gamma case, most easily obtained by first rewriting (4) as follows:

$$
\begin{aligned}
\widehat{U}(s) & =\frac{1}{v \log (1+s / \alpha)} \\
& =\frac{\alpha}{v s} \int_{0}^{1}\left(1+\frac{s}{\alpha}\right)^{u} \mathrm{~d} u \\
& =\frac{\alpha}{v} \int_{0}^{1}\left(\frac{1}{s} \frac{1}{(1+s / \alpha)^{1-u}}+\frac{1}{\alpha} \frac{1}{(1+s / \alpha)^{1-u}}\right) \mathrm{d} u,
\end{aligned}
$$

which implies, by [1, Equations (29.3.11), (29.2.6), and (6.5.2)], that

$$
u(t)=\frac{\alpha}{v} \int_{0}^{1} \frac{\mathrm{d} u}{\Gamma(u)}\left(\gamma(u, \alpha t)+(\alpha t)^{u-1} \mathrm{e}^{-\alpha t}\right),
$$

where $\gamma(u, t)=\int_{0}^{t} s^{u-1} \mathrm{e}^{-s} \mathrm{~d} s$ is the incomplete gamma function. We can also, by (11), obtain the following tail estimate:

$$
\mathrm{P}\left(\tau_{t}>x\right) \leq \exp \left(v x-\alpha t-x v \log \frac{v x}{\alpha t}\right)=\left(\frac{\alpha t}{v x}\right)^{v x} \mathrm{e}^{v x-\alpha t} .
$$

\section{Acknowledgements}

I am grateful to Jan Grandell for discussing Cox processes and renewal processes with me, and also to Thomas Höglund for a careful reading of an earlier draft of this paper and pointing out some errors. I also thank the anonymous referee, whose suggestions helped to improve the article.

\section{References}

[1] Abramowitz, M. and Stegun, I. A. (eds) (1992). Handbook of Mathematical Functions with Formulas, Graphs, and Mathematical Tables. Dover, New York.

[2] Bertoin, J. (1996). Lévy Processes. Cambridge University Press.

[3] Bertoin, J., van Harn, K. and Steutel, F. W. (1999). Renewal theory and level passage by subordinators. Statist. Prob. Lett. 45, 65-69.

[4] Bingham, N. H. (1971). Limit theorems for occupation times of Markov processes. Z. Wahrscheinlichkeitsth. 17, 1-22.

[5] Daley, D. J. and Vere-Jones, D. (2003). An Introduction to the Theory of Point Processes, Vol. 1, 2nd edn. Springer, New York

[6] Grandell, J. (1976). Doubly Stochastic Poisson Processes (Lecture Notes Math. 529). Springer, Berlin.

[7] KaJ, I. and Martin-Löf, A. (2004). Scaling limit results for the sum of many inverse Lévy subordinators. Submitted.

[8] Karatzas, I. And Shreve, S. E. (1988). Brownian Motion and Stochastic Calculus. Springer, New York.

[9] Kingman, J. F. C. (1964). On the doubly stochastic Poisson processes. Proc. Camb. Phil. Soc. 60, 923-930.

[10] Kozlova, M. and Salminen, P. (2004). Diffusion local time storage. Stoch. Process. Appl. 114, $211-229$.

[11] Sato, K.-I. (1999). Lévy Processes and Infinitely Divisible Distributions. Cambridge University Press.

[12] Steutel, F. W. and van Harn, K. (2004). Infinite Divisibility of Probability Distributions on the Real Line. Marcel Dekker, New York.

[13] Van Harn, K. and Steutel, F. W. (2001). Stationarity of delayed subordinators. Stoch. Models 17, 369-374.

[14] Winkel, M. (2005). Electronic foreign-exchange markets and passage events of independent subordinators. J. Appl. Prob. 42, 138-152. 